\title{
Effects of the non-perturbative mode structure on energetic particle transport
}

\author{
G. Meng ${ }^{1}$, Ph. Lauber ${ }^{1}$, Z. Lu ${ }^{1}$ and X. Wang ${ }^{1}$ \\ ${ }^{1}$ Max Planck Institut für Plasmaphysik, 85748 Garching, Germany \\ E-mail: guo.meng@ipp.mpg.de
}

5 March 2020

\begin{abstract}
Energetic particles (EPs) are known to change the properties of shear Alfvén waves in tokamaks, and for this reason non-perturbative models have to be used to describe their linear and non-linear evolution. In this work, mode structures distortion with symmetry breaking properties featured by a finite radial wave phase velocity due to EP effects are considered. A mode structure of the form $A(s)=$ $e^{-\sigma\left(s-s_{0}\right)^{2}}$ with complex parameters $\sigma$ and $s_{0}$ is used, to describe not only the width $(\propto 1 / \sqrt{\operatorname{Re}\{\sigma\}})$ and the mean location $\left(\operatorname{Re}\left\{s_{0}\right\}\right)$ of the bell-shaped radial structure, but also the radial phase variations $\left(\operatorname{Im}\{\sigma\}, \operatorname{Im}\left\{s_{0}\right\}\right)$, where $s$ is the normalized radial coordinate. The values of $\left(\sigma, s_{0}\right)$ are fitted from the results given by the gyrokinetic eigenvalue code LIGKA. The impact on EP transport is investigated using the driftkinetic code HAGIS. The effects of the mode structure symmetry breaking on the mode saturation level and the EP transport are analysed. In the presence of mode structure symmetry breaking which is relevant to the simulation and experimental observations, the growth rate as well as the particle and energy transport level can vary by $\sim 10 \%$. The velocity-space averaged parallel velocity of EPs in the inner region $s=0.2 \sim 0.5$ can change its sign for different mode structures, demonstrating the importance of the mode structure symmetry breaking on EP toroidal velocity reversal. This large effect $(\sim 100 \%)$ on the mean parallel flow could have implications for EP current drive and the transport in the burning plasmas.
\end{abstract}

Keywords: Energetic particle, transport, symmetry breaking 


\section{Introduction}

Energetic particle (EP) transport in the presence of Alfvénic modes determines the EP profiles and plays a crucial role in the overall plasma confinement. In order to model the EP transport efficiently, mixed linear-nonlinear recipes are used in various codes $[1,2,3]$. In this scheme, the linear mode structure and the (complex) mode frequency are determined with an eigenvalue solver, and then these linear properties are used in the calculation of the nonlinear wave-EP interaction and the consequent EP transport. For the perturbative approach, the linear mode structure is calculated without taking into account the EP or kinetic background species effects [4]. While the "perturbative" approach captures the dominant features of the instability excitation, the "non-perturbative" effects cause a "distortion" of the mode which has been generally observed by Electron Cyclotron Emission Imaging (ECEI) in various experiments $[5,6,7]$. It has been reported that EP can modify the Alfvén eigenmode structures with respect to the mode radial location and width [8], and can cause significant mode structure symmetry breaking [9]. Meanwhile, the mode frequency, as the eigen value of the linear system, or as a solution of the nonlinear dynamic system, can also change due to the EP effects. In turn, the non-perturbative mode structures are expected to bring the impacts on the linear growth rates, mode saturation levels and the EP transport $[10,11]$. These effects need to be evaluated based on comparative studies and therefore merit further investigation.

It is shown recently that the radial phase profile and velocity of the eigenmode can be modified by EP's non-perturbative effects, which causes the up-down asymmetry of the 2D mode structure [9]. A complex Gaussian representation of the mode structure capturing the symmetry breaking features in parallel and radial directions is analytically derived from the system of gyrokinetic equations [9]. In this work, we utilize this representation for the non-perturbative mode structure to study the induced EP transport. We use the HAGIS [12] code to study the effects of the asymmetric mode structures on the EP transport with the eigenmode solution calculated using LIGKA [13]. HAGIS models the nonlinear interaction between EPs described by drift-kinetic theory and a set of linear eigenmodes. It is assumed that the eigenmode structures do not change in HAGIS. The evolution of wave amplitude and the EPs distribution function are calculated according to the Lagrangian equation and the particles' equations of motion which describe the kinetic wave-particle nonlinearities. The total energy and canonical angular momentum of the wave-particle system is conserved in HAGIS. In addition, $\delta f$ method is adopted to reduce the numerical noise $[12,14]$.

We focus on the effects of mode symmetry breaking on the EP transport, especially the EP momentum transport. While symmetry breaking has been intensively studied in micro-turbulence transport (w/o EPs) due to its effects on intrinsic toroidal rotation $[15,16]$, its effects on EP transport are not studied to our knowledge. Thus, this work can also shed new light on intrinsic rotation studies for burning plasmas. This work is organized as follows. In Section 2, the representations of the mode structures with 
symmetry breaking for the EP transport studies are introduced. In Section 3, the simulation results of mode growth rate, saturation level and EP transport are shown and discussed. In Section 4, a summary and an outlook are given.

\section{Non-perturbative mode structure with Symmetry Breaking}

While the EP non-perturbative effects on the Alfvén modes have been discussed and analyzed theoretically [10, 9], this work focuses on the EP transport induced by the "nonperturbative" modes. The radial mode structures with different symmetry breaking features are represented by a complex Gaussian equation [9],

$$
\hat{\Phi}_{n m}(s)=e^{-\sigma\left(s-s_{0}\right)^{2}},
$$

where the radial coordinate $s=\sqrt{\psi}, \psi$ is the normalized poloidal flux, and $\sigma$ and $s_{0}$ are complex parameters. By solving the eigenvalue problem for Alfvén modes driven by EPs with trial functions of the form $\exp \left\{-\sigma\left(s-s_{0}\right)^{2}\right\}$ using the variational principle, it can be shown that $s_{0}$ and $\sigma$ are complex numbers whose imaginary parts are caused by the EPs contribution (details can be found in [9]). Here we define the complex radial wave vector as $k_{s}(s) \equiv-i d\left(\ln \hat{\Phi}_{n m}\right) / d s$, whose real part and imaginary part represent the radial variation of the phase and amplitude, respectively. With Eq. 1, we have

$$
k_{s}=-2\left[\left(s-s_{0, R}\right) \sigma_{I}-s_{0, I} \sigma_{R}\right]+2 i\left[\left(s-s_{0, R}\right) \sigma_{R}+s_{0, I} \sigma_{I}\right],
$$

where the subscript ' $\mathrm{R}$ ' and 'I' indicate the real and imaginary parts, respectively. While $1 / \sqrt{\sigma_{R}}$ and $s_{0, R}$ (in the third term) are linked to the width and the mean location of the Gaussian shape, $\sigma_{I}$ (in the first term) describes the $k_{s}$ component that has opposite signs in $s>s_{0, R}$ and $s<s_{0, R}$, and $s_{0, I}$ (in the second term) describes the $k_{s}$ component that is constant along $s$. Thus, the complex Gaussian functions in our work provide a simple but general way of representing the mode structure symmetry breaking. When we choose a complex $s_{0}$ but a real $\sigma$, i.e., $\sigma=\sigma_{R}$ (corresponding to case $\mathrm{C}$ in table 1 ), our complex Gaussian representation is consistent with the radially curved eigenmode wavefronts, as reported in the DIII-D studies [17].

To demonstrate the effect of mode symmetry breaking, we use a circular equilibrium with Grad-Shafranov shift with parameters matched to ASDEX-Upgrade, but the method is general and can be used for any equilibrium. The main parameters are as follows. Minor radius $a=0.496 \mathrm{~m}$, major radius $R_{0}=1.67 \mathrm{~m}$, magnetic field strength and safety factor at axis are $B_{0}=2.2 T$ and $q_{0}=1.99$ respectively. The minimum of $q$ is $q_{\min }=1.903$ at $s=0.494$. As the input of HAGIS, we use the Reversed Shear Alfvénic Eigenmode (RSAE) whose structure and frequency are calculated by LIGKA [13]. The mode structure and $q$ profile are shown in the left frame of figure 1 . The $q$ profile is very flat in the inner region. This RSAE has a toroidal mode number $n=2$, a dominant $m=4$ poloidal mode number and frequency $f_{n}=133 \mathrm{kHz}$. The parallel wave vector at $s=0.4$ is $k_{\|}(s=0.4) \approx(n q-m) /(q R)=-4.92 \times 10^{-4} / \mathrm{cm}$. When the mode structure is calculated using LIGKA, the ideal MHD model is adopted without the thermal ions kinetic effects and the EPs kinetic effects, serving as the base case. When 
the EPs kinetic effects are taken into account, the mode structure can be distorted significantly and the mode width and frequency also change. In this work, we focus on investigating the effects of mode structure symmetry breaking, so we use the same mode eigen frequency as the input of HAGIS in the simulations.

We fit the $\mathrm{m}=4$ mode structure given by LIGKA with the complex Gaussian equation $\Phi=A e^{-\sigma\left(s-s_{0}\right)^{2}}$ using the least-squares method. The fitting results are shown in the right frame of figure 1 with $\sigma=39.25+1.14 i$ and $s_{0}=0.40+0.0015 i$. The LIGKA mode structure is almost symmetric. The fitting parameter $A=148.66-18.17 i=$ $149.77 e^{-0.1216 i}$ is related to the mode amplitude and initial phase. The mode structure is an input for HAGIS [12]. Since the mode amplitude changes due to the waveparticle interaction and oscillates according to $e^{-i \omega_{n} t}$ in HAGIS, where $\omega_{n}=2 \pi f_{n}$ is the eigenfrequency, the parameter $A$ can be considered as a normalization factor.
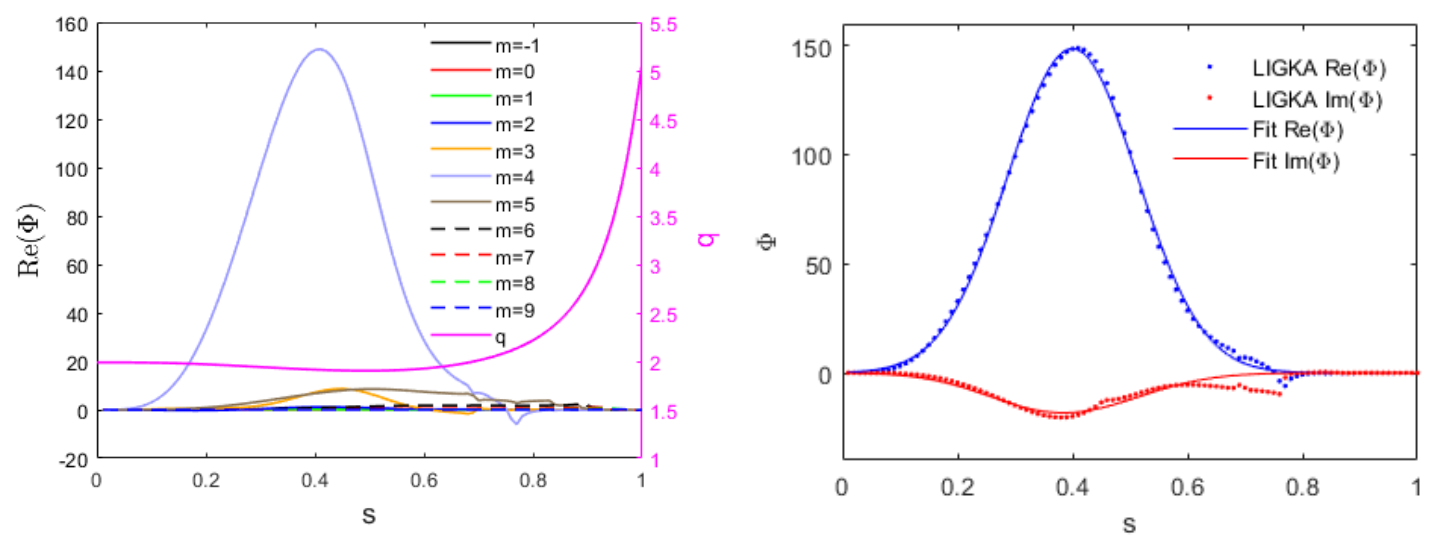

Figure 1. Left: the left axis corresponds to the radial mode structure calculated by LIGKA of the $n=2$ RSAE with the frequency $f=133 \mathrm{kHz}$ and the dominant harmonic $m=4$; the right axis corresponds to the safety factor $q$. The radial coordinate $s=\sqrt{\psi}$, where $\psi$ is the normalized poloidal flux. Right: the complex Gaussian fitting result of the $n=2, m=4$ eigenmode. The dotted lines and the solid lines indicate the LIGKA results and the fitted solutions respectively.

By adopting different values of the two parameters $\sigma$ and $s_{0}$, the mode structure can represent different symmetry breaking features. We choose four sets of representative parameters $\left(\sigma, s_{0}\right): \mathrm{A}, \mathrm{B}, \mathrm{C}$ and $\mathrm{D}$ as shown in table 1 . Case $\mathrm{A}$ is the base case with the symmetric mode structure and cases B, C and D mimic the experimental observations [17] and the HMGC simulation results [18]. The mode structures corresponding to

Table 1. Parameters $\sigma$ and $s_{0}$ choosen for four cases.

\begin{tabular}{l|l|l|l|l} 
& $\mathrm{A}$ & $\mathrm{B}$ & $\mathrm{C}$ & $\mathrm{D}$ \\
\hline$\sigma$ & 40 & $40-50 \mathrm{i}$ & 40 & $40-50 \mathrm{i}$ \\
\hline$s_{0}$ & 0.4 & 0.4 & $0.4+0.02 \mathrm{i}$ & $0.4+0.02 \mathrm{i}$
\end{tabular}

different values of $\sigma$ and $s_{0}$ are shown in figure 2. The radial wave vector, $k_{s}=i 2 \sigma\left(s-s_{0}\right)$, is zero when $\sigma$ and $s_{0}$ are both real. The real part of wave vector represents the 
wave radial propagation. Figure $2(\mathrm{~A})$ corresponds to the mode structure with no EP contribution ( $\sigma$ and $s_{0}$ both real), characterized with up-down symmetry [9]. Correspondingly, the mode does not propagate in the radial direction. Figure 2(B) corresponds to the boomerang like mode structure with real $s_{0}$ but complex $\sigma$. The wave propagates at the same speed but in opposite directions on both sides of $s_{0}$. Figure 2(C) corresponds to the mode structure with real $\sigma$ but complex $s_{0}$, and thus $\operatorname{Re}\left(k_{s}\right)=1.6$ as a constant number. The wave has a pure positive radial propagation with $k_{r}=k_{s} \partial s / \partial r \approx k_{s}(1 / 49.57) / \mathrm{cm}=0.032 / \mathrm{cm}$. For case $\mathrm{C}$, the mode is distorted clockwise as shown in the $2 \mathrm{D}$ mode structure in figure 2. If the $\operatorname{Im}\left(s_{0}\right)$ is negative, the mode is distorted counter-clockwise. Figure 2(D) corresponds to the mode structure with complex $\sigma$ and $s_{0}$, thus the wave vector $k_{s}$ is not constant and when $s=\operatorname{Re}\left(s_{0}\right)$, the wave vector is not zero, $k_{s}(s=0.4) \neq 0$. While the fitting procedure is based on the mode structure from LIGKA, it can be readily applied to treat the experimental ECEI data for further estimation of the non-perturbative mode structure effects.
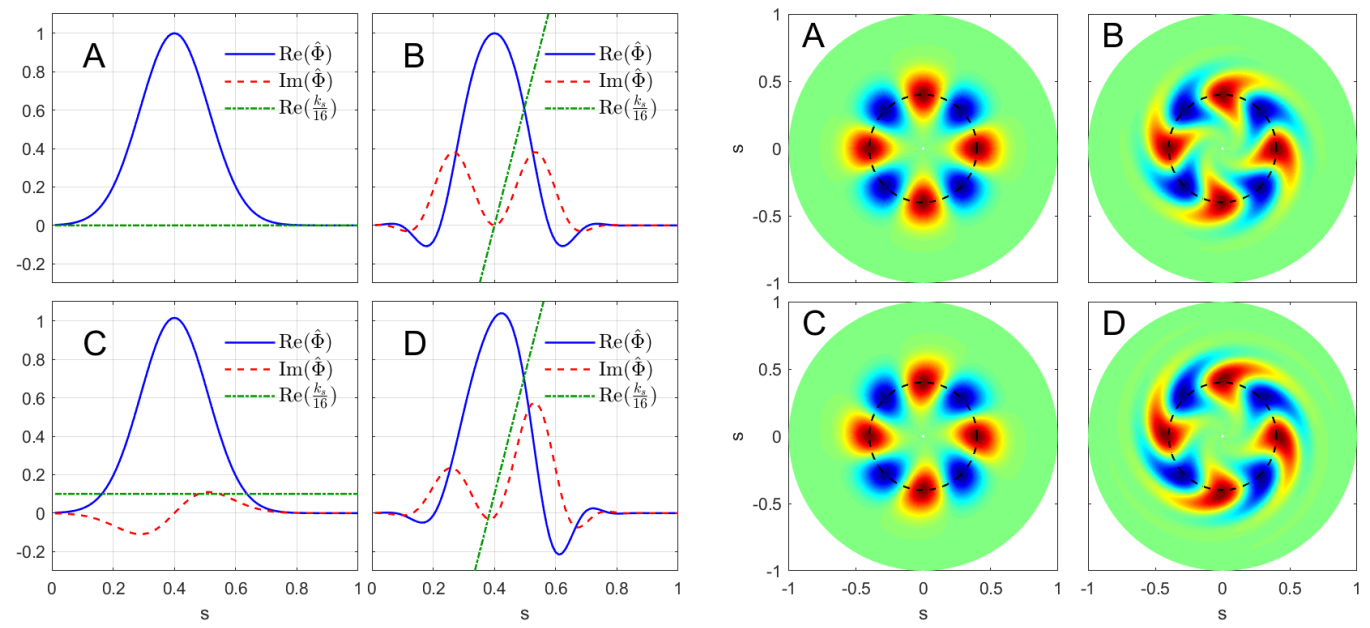

Figure 2. Four typical mode structures with different values of $\left(\sigma, s_{0}\right)$ listed in table 1. Left: The mode structures $\hat{\Phi}(s)$ and the wave vector $k_{s}$. Right: $2 \mathrm{D}$ mode structure.

\section{Simulation results}

The wave-EP nonlinearity and the related EP transport are simulated using the HAGIS code. The mode saturation is determined completely by the depletion of the free energy in the particle distribution function, since neither collisions nor turbulence is taken into account, and there is no source term for EPs. In HAGIS, each toroidal harmonic of a given mode with eigenvalue $\omega_{n}$ can be represented in the form,

$$
\widetilde{\Phi_{n}}=A_{n}(t) e^{-i \varpi_{n}(t)} \sum_{m} \hat{\Phi}_{n m}(s) e^{i\left(n \zeta-m \theta-\omega_{n} t\right)},
$$

where $n$ refers to the toroidal mode number of the single mode with angular frequency $\omega_{n}, m$ the poloidal harmonics, $A_{n}$ the real amplitude and $\varpi_{n}(t)$ the EP induced 
non-perturbative phase change (the change in amplitude and $\varpi_{n}(t)$ is much slower than the wave oscillation). The change of the wave frequency is $\delta \omega(t)=d \varpi_{n} / d t$. Here, $\zeta$ is the toroidal azimuthal angle and $\theta$ is the poloidal angle. The fixed linear eigenfunction $\hat{\Phi}_{n m}(s)$ can be a complex quantity [12]. Generally, for multiple harmonics, the symmetry breaking features such as $\exp \left\{-\sigma_{n m}\left(s-s_{0, n m}\right)^{2}\right\}$ of different harmonics can be different and can be included in $\hat{\Phi}_{n m}(s)$. In addition, by writing $\hat{\Phi}_{n m}(s)=\hat{A}_{n m}(s) \exp \left\{-\sigma_{n m}\left(s_{n m}-s_{0, n m}\right)^{2}\right\}$, the information regarding the relative phase-shifts between neighbouring harmonics $\hat{A}_{n m}(s)$ can be also included. In this work, we only keep one harmonic and $\hat{\Phi}(s)$ in each case contains different mode structure symmetry breaking features.

\subsection{Test Particle studies on Wave-Particle Resonance}

Generally, for a particle with given constants of motion, the linear wave-particle resonance condition is given by $n\left\langle\omega_{\zeta}\right\rangle-p\left\langle\omega_{\theta}\right\rangle=\omega_{n}$, where $p$ is an integer, $\omega_{\zeta}$ and $\omega_{\theta}$ are the toroidal and poloidal transit frequencies and $\langle\ldots\rangle$ denotes averaging over one poloidal transit time [19]. For low energy particles in a circular cross-section tokamak, the dominant resonance harmonics are $p=m \pm 1$ [20]. In strongly shaped plasmas and for large drift-orbit displacements, higher order harmonics are also important [21]. The exact resonance location can be only found by integrating over the particle poloidal trajectory [22]. According to quasi-linear theory, diffusion only occurs for the particles that exactly satisfy the resonance condition via a delta function [23]. However, the resonances are, in principle, broadened due to the finite growth rate, finite mode amplitude and the collisions [24, 25], and also effectively broadened due to stochastic processes affecting the resonant particles [26], such as collisions and microturbulence [27]. In addition, the mode structures are important for the wave-particle interactions. For example, a narrow structure can lead to a significant decrease in the resonance width compared to a case in which the eigenfunction does not vary across the resonant island [20] and the scaling of mode saturation levels is linear proportional to the initial growth rate, $A_{\text {sat }} \propto \gamma_{L}$ instead of $\propto \gamma_{L}^{2}[28,29]$.

To analyse the mode structure effects on the wave-particle resonances, a twodimensional scan has been performed in initial particle parallel velocity and initial radius $\left(v_{\|}, s\right)$, where $v_{\|}$is the parallel velocity. As the initial condition, the particle perpendicular velocity $v_{\perp}=0$, the particle location $\theta=0, \zeta=0$. In the test particle studies, particles are pushed in the field given by Eq. 3 without modification to the field due to EPs' feedback. The particles energy variation during 20 poloidal circles in the presence of a mode with the fixed amplitude $\delta B_{r} / B=10^{-3}$ ( $\approx$ HAGIS saturation value) and locked phase shift $\varpi=0$ is shown in figure 3 , where the Alfvén velocity $v_{A}$ is used for normalization, with $v_{A}=8.265 \times 10^{6} \mathrm{~m} / \mathrm{s}$. The corresponding initial energy range of the y-axis on figure 3 is from $15 \mathrm{keV}$ to $150 \mathrm{keV}$. The particle energy changes significantly at the $p=2$ resonance for s around 0.4 where the mode peaks. Resonance lines are visible in $\mathrm{A}, \mathrm{B}, \mathrm{C}$ and $\mathrm{D}$ and are distorted due to different mode structures. 


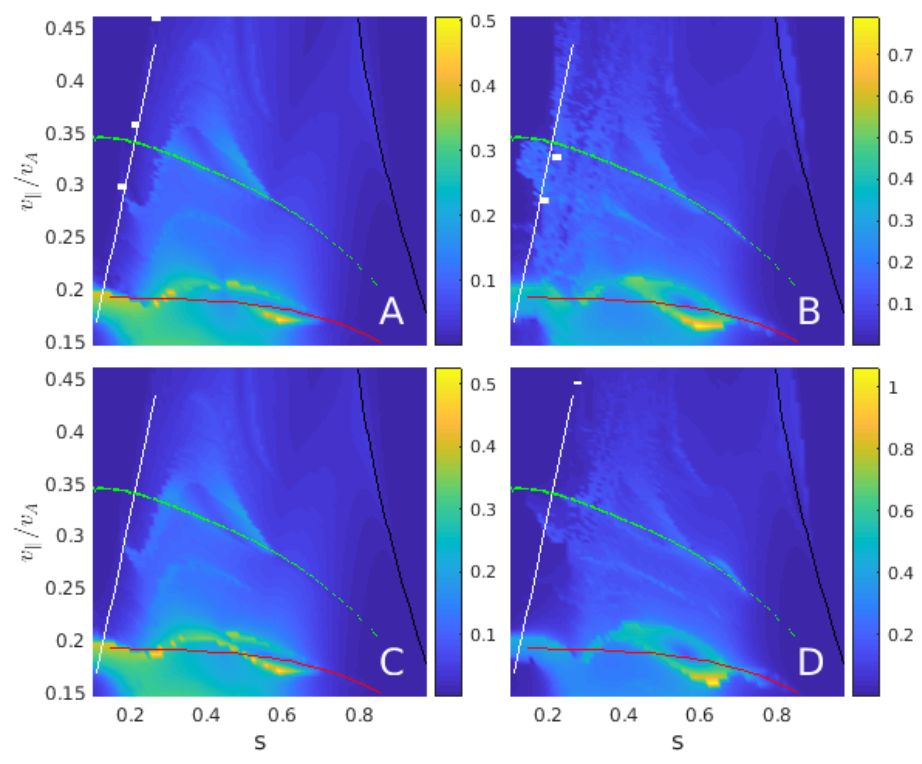

Figure 3. Fractional change in energy $|\Delta E| / E_{0}$ of the deeply passing $\left(v_{\|} / v=1\right)$ EPs arising from their interaction with the mode whose amplitude is fixed $\delta B_{r} / B=10^{-3}$ for four cases. The red line shows where linear resonance condition is satisfied, i.e. $n\left\langle\omega_{\zeta}\right\rangle-p\left\langle\omega_{\theta}\right\rangle=\omega_{n}$ with $p=2$. The black line corresponds to $p=3$ while the green line to $p=2.5$. Particles on the left side of the white line are with stagnation orbits (passing orbits that do not enclose the magnetic axis).

The resonance pattern and magnitude in $\mathrm{A}$ and $\mathrm{C}$ are similar; and those in $\mathrm{B}$ and $\mathrm{D}$ are similar (also see saturation level analyses following). For cases B and D, featured with a significant radial propagation, the resonance structures are distorted apparently compared with case A.

\section{2. $\delta f$ simulation of EP transport}

3.2.1. EP initial distribution. In order to study the nonlinear wave-particle interaction and the consequent EP transport, the $\delta f$ simulation is performed using HAGIS. The radial distribution of the $\mathrm{EP}$ density is assumed to be

$$
f(\psi)=\frac{1}{1+\exp \left(\frac{\psi-\psi_{0}}{\delta \psi}\right)},
$$

with $\psi_{0}=0.16$ and $\delta \psi=0.2$. The EP density at magnetic axis is $n(0)=9.163 \times$ $10^{17} \mathrm{~m}^{-3}$. We use a slowing down distribution in energy $(E)$,

$$
f(E)=\frac{1}{E^{3 / 2}+E_{c}^{3 / 2}} \operatorname{erfc}\left(\frac{E-E_{0}}{\Delta E}\right),
$$

with a birth energy of $E_{0}=93 \mathrm{keV}, E_{c}=37.21 \mathrm{keV}, \Delta E=149.9 \mathrm{keV}$. The beta of EP population is $\beta_{f}=0.1 \%$. We use an isotropic pitch angle distribution. In the following simulations, we use 300000 markers, loading position $s \in[0.001,0.9]$ and energy range $E \in[10 \mathrm{keV}, 100 \mathrm{keV}]$. 
3.2.2. Mode saturation and linear growth rate. The linear growth rate $\gamma_{L}$ is fitted between the 200 th to 1000 th steps $\left(1\right.$ step $\left.=3.7678 \times 10^{-7} \mathrm{~s}\right)$. The saturation level $A_{\text {sat }}$ and the frequency change of the mode $\delta \omega$ are averaged over time window 10000th15000th steps $(t=3.77-5.65 \mathrm{~ms})$ as shown in figure 4(a). The linear growth rate, the saturation level and the frequency change of base case $\mathrm{A}$ (symmetric) are $\gamma_{L} / \omega_{n}=1.00 \times 10^{-2}, A_{\text {sat }}=3.61 \times 10^{-3}$ and $\delta \omega / \omega_{n}=-1.64 \times 10^{-2}$. These values of the base case $A$ are used as the units of the linear growth rate, the saturation level and the $\delta \omega$, and other cases are always compared with the base case A, as shown in figure 4(b). For Case B compared with Case A, the linear growth rate and saturation level decrease by $10 \%$ and $20 \%$ respectively due to mode structure distortion. For Case D, $A_{\text {sat }}$ and $\gamma_{L}$ decrease compared with Case A, which shows the similar trend as Case B. For Case C, $\gamma_{L}$ and $A_{\text {sat }}$ slightly change compared with Case A. The relative difference of frequency change, $\delta \omega / \omega_{n}$, in B, C and D compared with Case A can be $\sim 20 \%$.
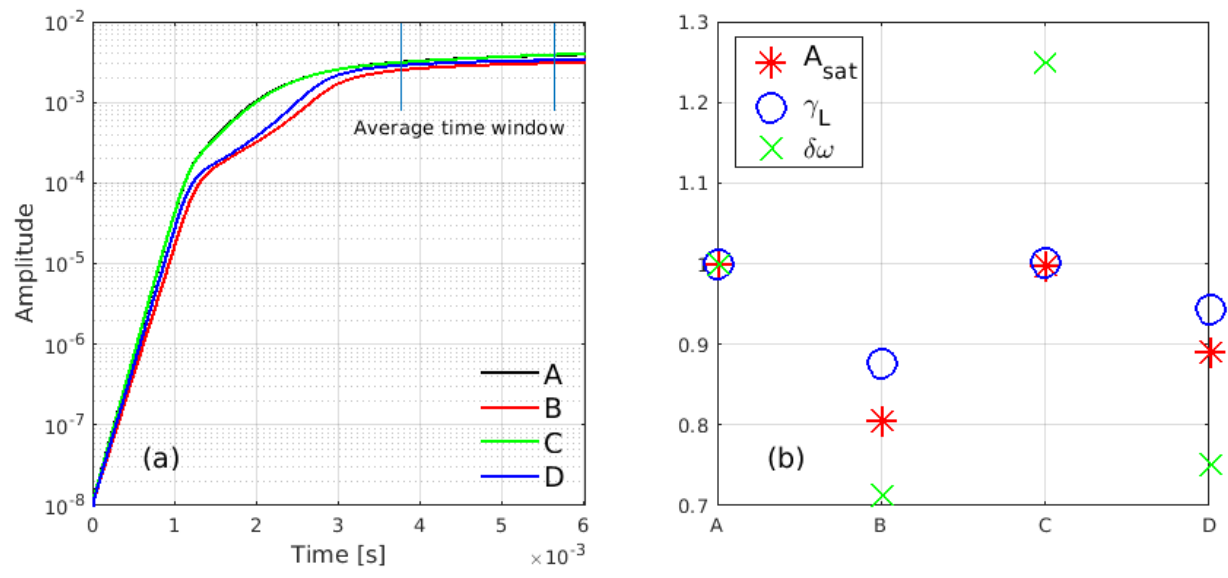

Figure 4. (a) Mode amplitude variation with time for A, B, C and D four cases. (b) Mode linear growth rate, saturation level, frequency change at saturation, compared with base case $\mathrm{A}$.

3.2.3. Particle redistribution at saturation. The flattening of the density and energy profiles induced by the Alfvén modes occurs when the resonance condition is met, as shown in the left and middle frames of figure $5(\delta n, \delta E<0$ for $s<0.5 ; \delta n, \delta E>0$ for $s>0.5)$. The radial distribution is obtained by integrating over velocity space and the other real space coordinates. Perturbative mode structures (B, C, D) lead to changes in particle and energy transport with $\delta n, \delta E$ deviating by $\sim 10 \%$. In contrast, the parallel velocity profile changes significantly due to the non-perturbative mode structure symmetry breaking as shown in right frame of figure 5 . In the inner region $(s<0.5), u_{\|}$can even change its direction (rotation reversal). The average is taken during $\mathrm{t}=3.77-5.65 \mathrm{~ms}$ (at saturation) in all figures. The radial profiles of the perturbed EP density (left), energy (middle) and parallel (right) velocity are calculated according to $\delta n=\int \delta f d v^{3} / \int f d v^{3}, \delta E=\int \delta f \cdot E d v^{3} / \int f d v^{3}$, and $u_{\|}=\int \delta f \cdot v_{\|} d v^{3} / \int f d v^{3}$ 
respectively. Note that for case $\mathrm{C}$ (with real $\sigma=40$ and complex $s_{0}=0.4+0.02 i$ ), the mode is only slightly distorted and its saturation level, linear growth rate and $\delta \omega$ are all similar to case A. However, the $u_{\|}$direction in the inner region $(s<0.5)$ is opposite with that in cases $\mathrm{B}$ and $\mathrm{D}$. This demonstrates the importance of the mode structure symmetry breaking for the EP toroidal momentum transport, in particular in the case of unidirectional or, more generally, asymmetric radial propagation.
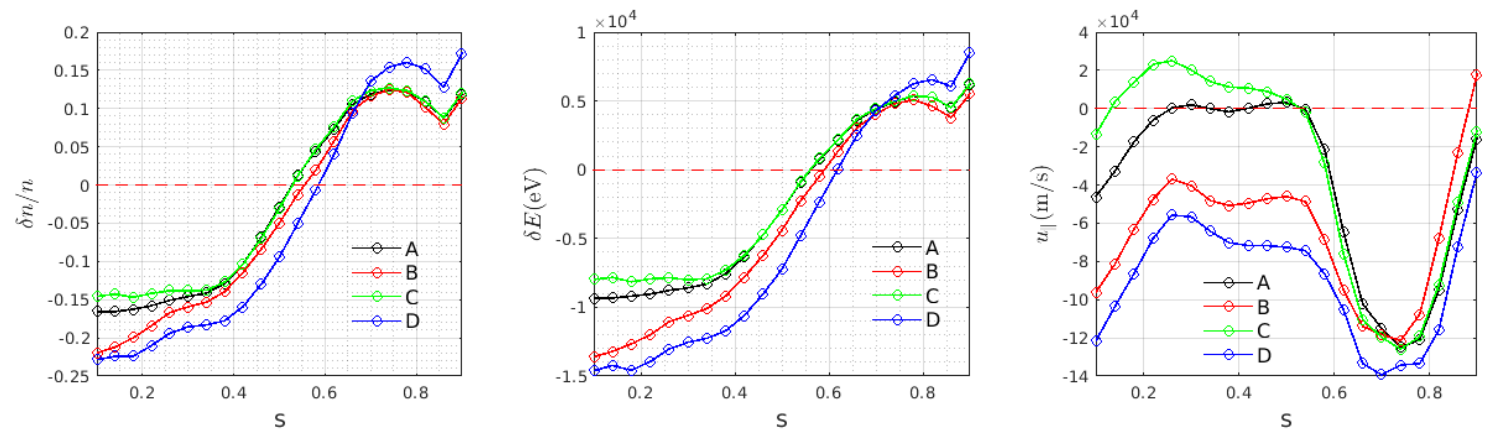

Figure 5. The radial profile of the perturbed EP density (left), energy (middle) and the parallel velocity (right) at saturation (averaged during $t=3.77-5.65 \mathrm{~ms}$ ). The integration is performed over velocity space and other configuration space coordinates in obtaining the radial profile.

3.2.4. $u_{\|}$reversal due to the mode radial wave vector. The reversal of the $u_{\|}$for different mode structures is investigated in more detail by further modification of case C. As shown in figure 7 , the variation of $\operatorname{Im}\left(s_{0}\right)$ leads to the significant change of the EP parallel velocity $u_{\|}$. For these cases, $\sigma$ is kept real with $\sigma=40$. A $u_{\|}$reversal in the inner region is observed as $\operatorname{Im}\left(s_{0}\right)$ varies, and the $u_{\|}$of EPs at $s=0.26$ can be produced with a magnitude of $\sim 10 \mathrm{~km} / \mathrm{s}$. For these $u_{\|}$reversal cases, the mode distortions are relatively small $\left(\left|\operatorname{Im}\left(s_{0}\right)\right| \leq 0.03\right)$. The mode saturation level and the linear growth rate deviate from case $\mathrm{A}\left(s_{0}=0.4\right)$ by less than $6 \%$ as shown in figure 6 . The frequency change of the mode at saturation $\delta \omega / \omega_{n}$ varies from $-0.88 \times 10^{-2}$ to $-2.13 \times 10^{-2}$ for $\operatorname{Im}\left(s_{0}\right)$ varying from $-0.03 i$ to $0.03 i$. As shown in figure 8 , the density and energy profiles of these cases change mainly at $s<0.4$ region and less than $20 \%$ compared with the base case $\left(s_{0}=0.4\right)$. We also did a scan for varying $\operatorname{Im}(\sigma)$ and keeping $s_{0}=0.4$, the $u_{\|}$does not change direction, emphasising that $\operatorname{Im}\left(s_{0}\right)$ is the key parameter causing $u_{\|}$reversal.

3.2.5. Generation of $u_{\|}$due to the asymmetric wave-particle interaction. The perturbed distribution $\delta f$ in velocity and real space is studied to analyse the $u_{\|}$ generation. The $\delta f$ along pitch angle and radial directions, $\delta f(\lambda, s)$, is shown in figure 9 for negative and positive $\operatorname{Im}\left(s_{0}\right)$ cases, where $\lambda=v_{\|} / v$. For two different cases with $s_{0}=0.4-0.03 i$ (left) and $s_{0}=0.4+0.03 i$ (right), the change of the $\delta f$ value is more visible for the co-moving particles in the $s<0.4$ region (top left corner) as shown in 

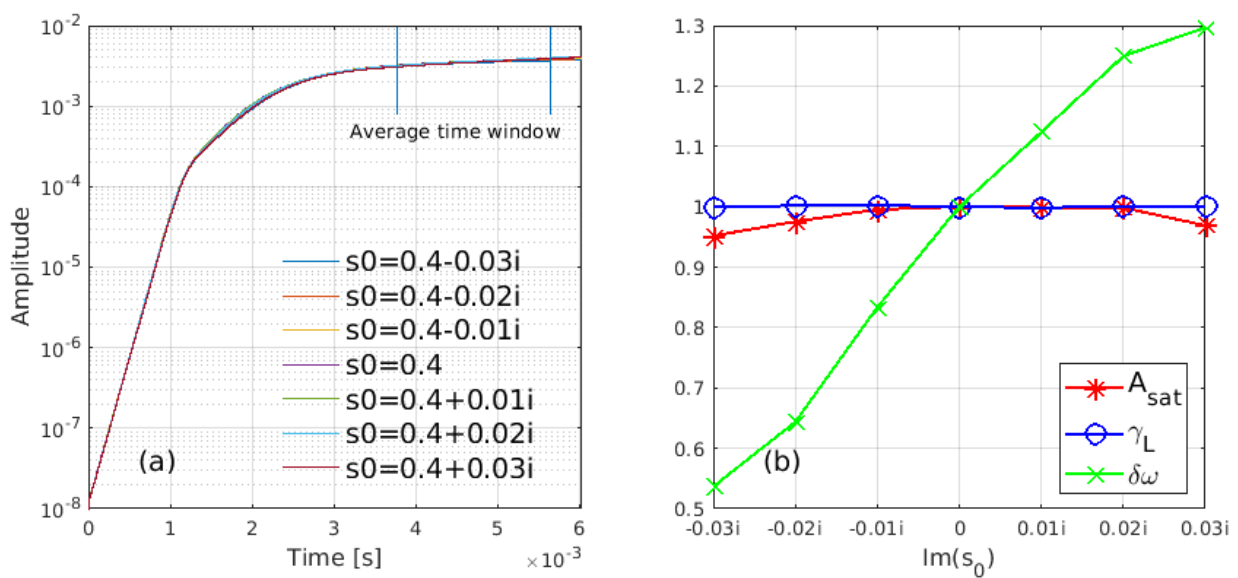

Figure 6. (a) Mode amplitude variation with time for the $u_{\|}$reversal cases. (b) The mode linear growth rate, saturation level, frequency change at saturation, compared with case A. The frequency change of the mode at saturation $\delta \omega / \omega_{n}$ varies from $-0.88 \times 10^{-2}$ to $-2.13 \times 10^{-2}$ for $\operatorname{Im}\left(s_{0}\right)$ varying from $-0.03 i$ to $0.03 i$.
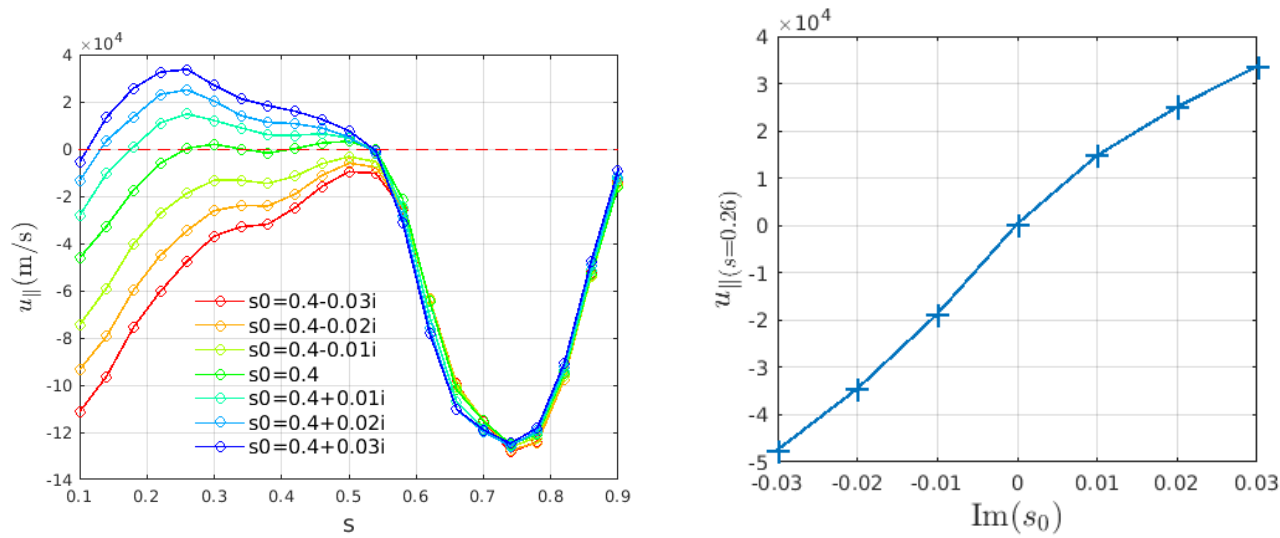

Figure 7. Left: EP parallel velocity radial distribution $u_{\|}(s)$ changes with $s_{0}$. The $u_{\|}$in the inner region changes direction with $\operatorname{Im}\left(s_{0}\right)$. Right: $u_{\|}(s=0.26)$ varies with $\operatorname{Im}\left(s_{0}\right)$.
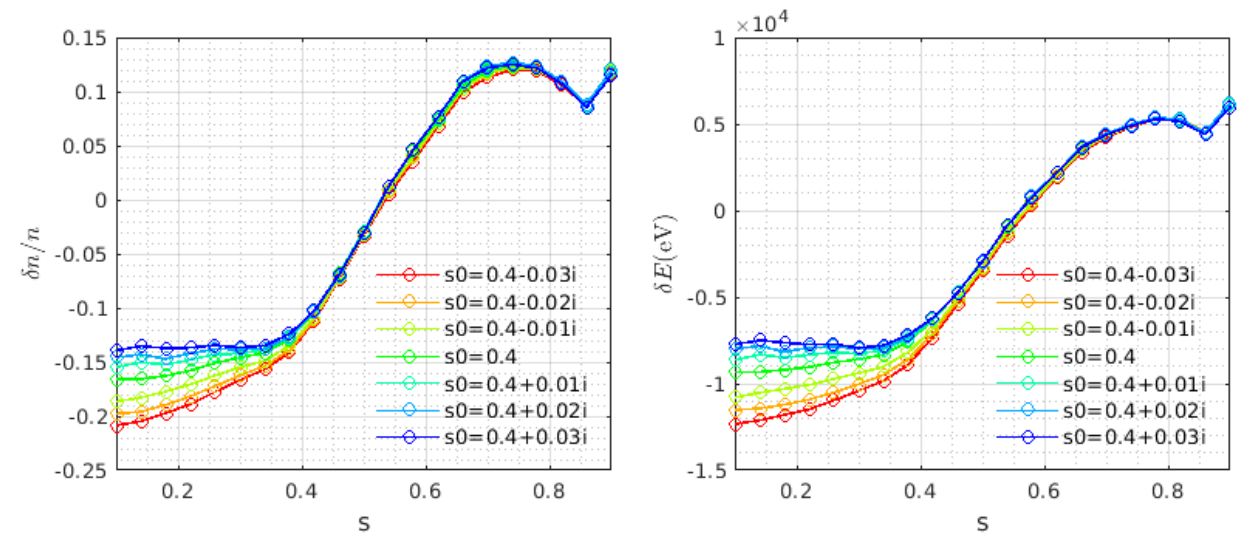

Figure 8. Radial profile of the density and $\delta E$ at saturation. 

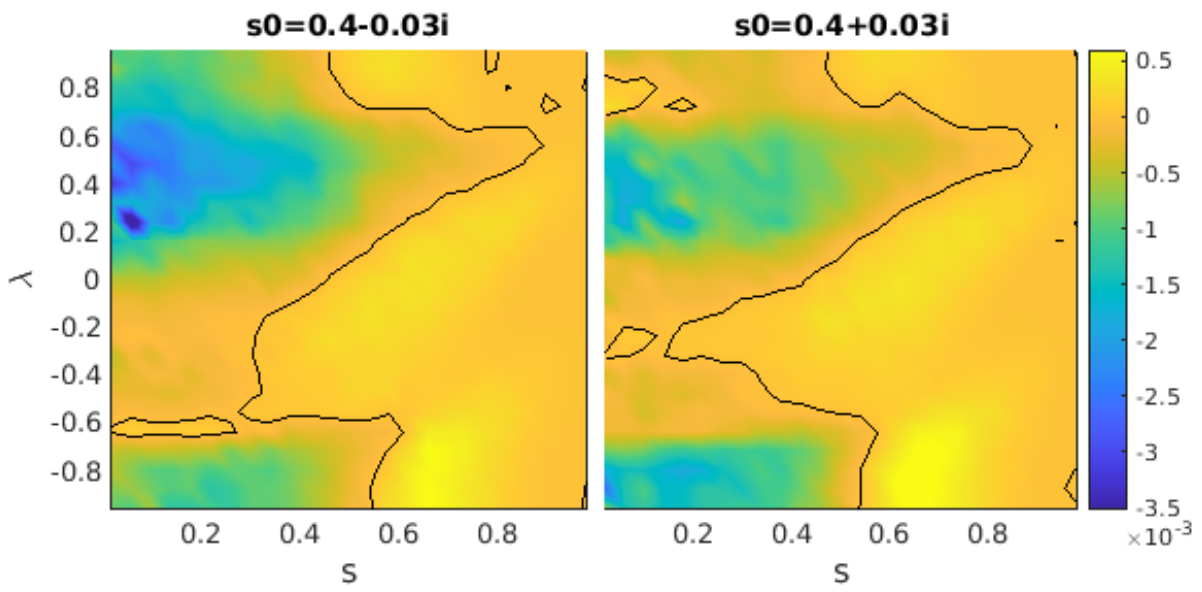

Figure 9. $\delta f(\lambda, s)$ of EPs for negative and positive $\operatorname{Im}\left(s_{0}\right)$, where $\lambda=v_{\|} / v$. The black line is the contour of $\delta f=0$.

figure 9, which is consistent with the results in figure 8. The perturbed distribution $\delta f$ averaged over $s \in[0.2,0.4]$ and along $\lambda$ is shown in figure 10(a). The responses of particles are different according to their pitch angles. $\delta f$ of co-moving particles are more sensitive to the $\operatorname{Im}\left(s_{0}\right)$. Particles with $\lambda \in[0.3,1]$ response more strongly and as $\operatorname{Im}\left(s_{0}\right)$ changes, $\delta f$ near $\lambda=0.5$ changes significantly. The response region of $\delta f$ for counter-moving particles is much narrower, mainly in $\lambda \in[-1,-0.6]$.
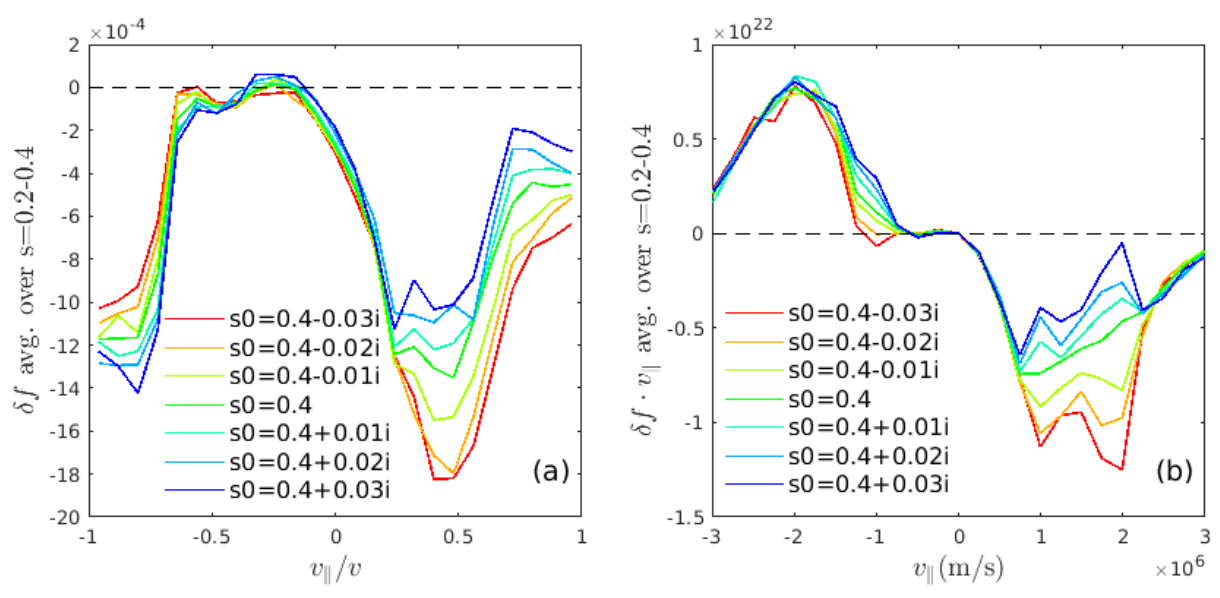

Figure 10. (a) $\delta f\left(v_{\|} / v\right)$ of EPs averaged over $\mathrm{s}=0.2-0.4$. (b) Asymmetric $\delta f \cdot v_{\|}$ distribution of EPs averaged over $\mathrm{s}=0.2-0.4$.

The $\delta f \cdot v_{\|}$distribution along $v_{\|}$is shown in figure 10(b). The contributions of co-moving particles are negative and the contributions of counter-moving particles are positive. When the $\operatorname{Im}\left(s_{0}\right)$ changes from negative to positive values, the contributions of counter-moving particles increase and the contributions of co-moving particles decrease, leading to the generation of the net parallel velocity. While the asymmetric particle response of thermal ions to the microturbulence has been studied in previous work [30], 
our work shows that for EP transport, the asymmetric mode structure also plays an important role in particle response and the $u_{\|}$generation.

Since the total toroidal angular momentum of the wave-particle system is conserved for any isolated system, the toroidal momentum evolution equation can be written in conservative form $[15,31,32], \partial_{t} P_{\phi}+\partial_{t} g_{\phi}+\nabla \cdot \Pi_{\phi}=0$ where $P_{\phi}$ is the particles toroidal momentum, $g_{\phi}$ is the toroidal component of the electromagnetic field momentum, and $\Pi_{\phi}$ is the toroidal momentum flux. The momentum density $\vec{g}$ of the Alfvén wave can be written explicitly, $\vec{g}=\varepsilon_{0} \delta \vec{E} \times \delta \vec{B}=\vec{B} /(2 B)\left\{\left(k_{\|} / \omega\right)\left(k_{r R}^{2}+k_{\theta}^{2}+k_{r I}^{2}\right)\left|\hat{\Phi}_{n m}\right|^{2}\right\}$, where $\varepsilon_{0}$ is the vacuum permittivity, the subscripts $R$ and $I$ indicate the real and imaginary parts,

$k_{r}=k_{s} \partial s / \partial r, k_{\theta}=m / r$. For the Alfvén eigenmode, $E_{\|}=-(\vec{B} / B) \cdot \nabla \hat{\Phi}-\partial \hat{A}_{\|} / \partial t=0$ approximation has been adopted, where $\hat{A}_{\|}$is the parallel component of the vector potential. In this work, $k_{\theta}=0.263 / \mathrm{cm}, k_{r R}=0.032 / \mathrm{cm} \ll k_{\theta}$ at $s=0.4$ for Case C with $s_{0}=0.4+0.02 i$, the magnitude of wave momentum $\vec{g}$ changes very little when determining the dependence of the $u_{\|}$reversal on $\operatorname{Im}\left(s_{0}\right)$. This becomes obvious when comparing mode with positive and negative values of $\operatorname{Im}\left(s_{0}\right)$ : the wave momentum $\vec{g}$ is the same but the $u_{\|}$in the inner region is opposite, as shown in figure 7 . Thus although the direct momentum source from the wave could contribute to the $u_{\|}$, other mechanisms need to be considered to uncover the $u_{\|}$generation. The complete form of the Reynolds-Maxwell stress $\left(\Pi_{\phi}\right)[31,32]$ needs to be derived with the consideration of necessary effects such as the polarization drift effect [33], the turbulence acceleration mechanism [34] and the EP physics such as the finite Larmor radius/finite orbit width effect [35].

As is mentioned, the perturbative approach implemented in HAGIS conserves the total energy and toroidal momentum, but the mode structure is fixed $[12,14]$. Thus the difference between transients and persistent processes of the $u_{\|}$generation related to the mode structure time variation cannot be distinguished. In a more self-consistent nonlinear simulation with mode structure modification, the radially varying wave phase may change in time and thus generate a different time-averaged $u_{\|}$profile. While the toroidal momentum transport can be transient, the nonlinear balance can be attained and the eventual mode structure and $u_{\|}$can differ from the transient ones. Nevertheless, the mode structure observed in experiment and simulation shows that radially varying $\mathrm{AE}$ phase fronts can persist during nonlinear saturation phase $[5,18]$ and thus, a finite level of the $u_{\|}$distribution is expected. To estimate this non-linear balance quantitatively, more self-consistent simulations using gyrokinetic codes will be necessary for a more accurate calculation of the $u_{\|}$generation.

\section{Summary}

The effects of the non-perturbative mode structure on the energetic particle transport have been analyzed in this work. The LIGKA-HAGIS [13, 12, 3] coupling scheme has been applied to investigate the EP-wave interaction and the EP transport. In the simulations, the mode structures are fitted using the analytical formula with 
symmetry breaking properties based on Ref. [9] in order to mimic the experimental and simulation observations [5, 8]. Analyses based on ASDEX-Upgrade parameters show that non-perturbative mode structure can be important for EP transport modelling. The particle resonance changes and the wave-particle resonance island structures are distorted due to the mode structure symmetry breaking. The mode radial propagation (asymmetry of radial wave vector $k_{s}$, caused by radial non-uniformity with respect to mode rational surface) changes the EP transport, especially the toroidal momentum transport. Specifically, with mode symmetry breaking effects considered, the mode linear growth rate can change by $10 \%$ and the saturation level can change by $20 \%$. While the EP density and energy transport can change by of order 10\%, the EP parallel velocity distribution can change significantly, with the magnitude of $\sim 10 \mathrm{~km} / \mathrm{s}$. Especially, $u_{\|}=\delta f \cdot v_{\|}$radial profile reversal in the inner region is observed when varying the mode structures. This demonstrates the feature of toroidal momentum transport in the presence of EPs, as a complement to the momentum transport studies for bulk plasmas $[16,15,36,37]$. It is identified that the non-perturbative treatment is important, especially for the studies of the EP intrinsic rotation and the parallel EP current formation. The simulation stresses the importance of global analysis, however, based on [9], the global effects can be included in local (and thus cheap) analysis. As a result, this work also opens up the possibility for non-perturbative EP transport analyses in experiments when coupled to 2D mode structure measurement such as ECEI diagnostic. In addition, self-consistent nonlinear electromagnetic simulations using gyrokinetic or hybrid approaches $[8,38,39,40]$ can include more comprehensive physics ingredients in order to reveal more details of the EP physics, such as phase space zonal structure dynamics [41, 42]. Specifically, the following aspects merit more effort in order to build our capability of predicting the EP transport, especially the parallel momentum transport.

- While in this work shows that the EP transport can be affected due to the mode structure distortion with reasonably selected parameters $\left(\sigma, s_{0}\right)$, the hybrid and gyrokinetic simulations are needed to identify the quantitative connection between $\left(\sigma, s_{0}\right)$ and the EP profiles such as the maximum EP drive location and the nonlinear phenomenon such as frequency chirping that are observed in hybrid and gyrokinetic simulations $[43,44]$. With this quantitative mapping between EP parameters, mode distortion parameters $\left(\sigma, s_{0}\right)$ and the EP transport modifications, the capability of interpreting and predicting the EP transport in experiments can be enhanced.

- In the self-consistent hybrid and gyrokinetic simulations, the mode frequency, growth/damping rate and the mode structure can change due to the mode-particle interaction [45]. The nonlinear effects on the EP transport, especially the $u_{\|}$ generation also needs to be evaluated. Our analyses also need to be extended to consider the nonlinear wave-wave and wave-particle effects on mode structure symmetry breaking and EP transport, by making use of various theoretical tools and transport theory $[46,25,37,15]$. 
These studies require dedicated effort and will be part of our future work for the study of the non-perturbative mode structure effects on EP transport.

\section{Acknowledgments}

The authors would like to thank Dr. F. Zonca, Dr. S.D. Pinches, Dr. B. Scott and T. Hayward-Schneider for fruitful discussions, partially within the EUROFUSION Enabling Research Projects Projects "NLED" (ER15-ENEA-03), "NAT" (CfP-AWP17ENR-MPG-01) and "MET" (ENR-MFE19-ENEA-05). This work has been carried out within the framework of the Eurofusion Consortium and has received funding from the Euratom research and training programme 2014-2018 and 2019-2020 under grant agreement No 633053. The views and opinions expressed herein do not necessarily reflect those of the European Commission.

\section{References}

[1] Gorelenkov N, Duarte V, Podesta M and Berk H 2018 Nuclear Fusion 58082016

[2] Bass E M and Waltz R 2017 Physics of Plasmas 24122302

[3] Schneller M, Lauber P, Brüdgam M, Pinches S D and Günter S 2012 Nuclear Fusion 52103019

[4] Chen L, White R and Rosenbluth M 1984 Physical Review Letters 521122

[5] Tobias B, Classen I, Domier C, Heidbrink W, Luhmann Jr N, Nazikian R, Park H, Spong D A and Van Zeeland M 2011 Physical Review Letters 106075003

[6] Classen I, Boom J, Suttrop W, Schmid E, Tobias B, Domier C, Luhmann Jr N, Donné A, Jaspers R, de Vries P et al. 2010 Review of Scientific Instruments 81 10D929

[7] Classen I, Lauber P, Curran D, Boom J, Tobias B, Domier C, Luhmann Jr N, Park H, Munoz M G, Geiger B et al. 2011 Plasma Physics and Controlled Fusion 53124018

[8] Wang Z, Lin Z, Holod I, Heidbrink W, Tobias B, Van Zeeland M, Austin M E et al. 2013 Physical Review Letters 111145003

[9] Lu Z X, Wang X, Lauber P and Zonca F 2018 Nuclear Fusion 5882021

[10] Zonca F, Briguglio S, Chen L, Fogaccia G and Vlad G 2005 Nuclear Fusion $45477-484$

[11] Chen L and Zonca F 2016 Reviews of Modern Physics 88015008

[12] Pinches S, Appel L, Candy J, Sharapov S, Berk H, Borba D, Breizman B, Hender T, Hopcraft K, Huysmans G et al. 1998 Computer Physics Communications 111 133-149

[13] Lauber P, Günter S, Könies A and Pinches S 2007 Journal of Computational Physics 226 447-465

[14] Pinches S D 1998 Nonlinear interaction of fast particles with Alfvén waves in tokamaks Ph.D. thesis University Nottingham

[15] Peeters A, Angioni C, Bortolon A, Camenen Y, Casson F J, Duval B, Fiederspiel L, Hornsby W, Idomura Y, Hein T et al. 2011 Nuclear Fusion 51094027

[16] Diamond P, McDevitt C, Gürcan Ö D, Hahm T, Wang W, Yoon E, Holod I, Lin Z, Naulin V and Singh R 2009 Nuclear Fusion 49045002

[17] Kramer G J, Tobias B J, Turnbull A and Bass E M 2019 Nuclear Fusion 59094001

[18] Briguglio S, Vlad G, Zonca F and Kar C 1995 Physics of Plasmas 2 3711-3723

[19] Berk H, Breizman B, Fitzpatrick J, Pekker M, Wong H V and Wong K 1996 Physics of Plasmas 3 1827-1838

[20] White R, Gorelenkov N, Duarte V and Berk H 2018 Physics of Plasmas 25102504

[21] Heidbrink W 2008 Physics of Plasmas 15055501

[22] White R B 2013 The theory of toroidally confined plasmas (World Scientific Publishing Co Inc)

[23] Kaufman A N 1972 The Physics of Fluids 15 1063-1069 
[24] Berk H, Breizman B, Fitzpatrick J and Wong H 1995 Nuclear fusion 351661

[25] Meng G, Gorelenkov N, Duarte V, Berk H, White R and Wang X 2018 Nuclear Fusion 58082017

[26] Ghantous K, Berk H and Gorelenkov N 2014 Physics of Plasmas 21032119

[27] White R, Duarte V, Gorelenkov N and Meng G 2019 Physics of Plasmas 26032508

[28] Wang X, Briguglio S, Lauber P, Fusco V and Zonca F 2016 Physics of Plasmas 23012514

[29] Briguglio S, Schneller M, Wang X, Di Troia C, Hayward-Schneider T, Fusco V, Vlad G and Fogaccia G 2017 Nuclear Fusion 5772001

[30] Wang W, Diamond P, Hahm T, Ethier S, Rewoldt G and Tang W 2010 Phys. Plasmas 17072511

[31] Scott B and Smirnov J 2010 Physics of Plasmas 17112302

[32] Abiteboul J, Garbet X, Grandgirard V, Allfrey S, Ghendrih P, Latu G, Sarazin Y and Strugarek A 2011 Physics of Plasmas 18082503

[33] McDevitt C, Diamond P, Gürcan Ö D and Hahm T 2009 Physical review letters 103205003

[34] Garbet X, Esteve D, Sarazin Y, Abiteboul J, Bourdelle C, Dif-Pradalier G, Ghendrih P, Grandgirard V, Latu G and Smolyakov A 2013 Physics of Plasmas 20072502

[35] Lauber P and Lu Z 2018 Analytical finite-lamor-radius and finite-orbit-width model for the ligka code and its application to kgam and shear alfvén physics Journal of Physics: Conference Series vol 1125 (IOP Publishing) p 012015

[36] Lu Z, Wang W, Diamond P, Tynan G, Ethier S, Chen J, Gao C and Rice J 2015 Nuclear Fusion 55093012

[37] Angioni C, Camenen Y, Casson F, Fable E, McDermott R, Peeters A and Rice J 2012 Nuclear Fusion $\mathbf{5 2} 114003$

[38] Lanti E, Ohana N, Tronko N, Hayward-Schneider T, Bottino A, McMillan B, Mishchenko A, Scheinberg A, Biancalani A, Angelino P et al. 2019 Computer Physics Communications

[39] Taimourzadeh S, Bass E, Chen Y, Collins C, Gorelenkov N, Könies A, Lu Z, Spong D, Todo Y, Austin M et al. 2019 Nuclear Fusion 59066006

[40] Lu Z, Lauber P, Hayward-Schneider T, Bottino A and Hoelzl M 2019 Physics of Plasmas 26 122503

[41] Zonca F, Chen L, Briguglio S, Fogaccia G, Vlad G and Wang X 2015 New Journal of Physics 17 013052

[42] Falessi M V and Zonca F 2019 Physics of Plasmas 26022305

[43] Wang X, Briguglio S, Chen L, Di Troia C, Fogaccia G, Vlad G and Zonca F 2012 Physical Review E 86045401

[44] Zhang H, Lin Z, Holod I, Wang X, Xiao Y and Zhang W 2010 Physics of Plasmas 17112505

[45] Briguglio S, Zonca F and Vlad G 1998 Phys. Plasmas 53287

[46] Briguglio S, Wang X, Zonca F, Vlad G, Fogaccia G, Di Troia C and Fusco V 2014 Physics of Plasmas 21112301 